\title{
Feature frequency extraction algorithm based on matching pursuit and its application in axis orbit
}

\author{
Weiguang $\mathrm{Li}^{1}{ }^{*}$, Xinxin Zhang ${ }^{1}$, Zhen $\mathrm{Li}^{1}$, Jian Huang ${ }^{1}$, Wenwei Cai ${ }^{1}$ \\ ${ }^{1}$ School of Mechanical and Automotive Engineering, South China University of Technology, Guangzhou 510640
}

\begin{abstract}
Rotor displacement signal is an important state characteristic parameter of rotating machinery, which contains abundant operation information of rotating machinery. Aiming at the problem of purifying axis orbit in large -scale rotor test-bed, a new method of extracting the characteristic frequency and synthesizing axis orbit is proposed based on the matching pursuit algorithm (MP). In this paper, the sparse decomposition of signals in the algorithm is implemented by MP algorithm. The algorithm can accurately extract single or multiple feature frequencies. The purified curve is clearer and more efficient. Fault types can be found by extracting different characteristic frequency synthetic axis trajectories, so as to understand the operation status of the large-scale rotor test-bed.
\end{abstract}

\section{Introduction}

In the process of manipulation monitoring and fault diagnosing in rotating machinery, the center position of rotary parts, that is axis orbit, contains massive information, which is of great significance to determining the rotor's running state and fault symptom [1-3]. By identifying the axis orbit and analyzing the operation status of the equipment, the early warning information of the fault can be obtained in advance[4, 5]. Recently, many scholars have done a lot of researches on the purification of axis orbit [6-10]. Ref. [6, 7] subdivided random frequency band infinitely via harmonic wavelet to extract frequency of Interest. The method of empirical mode decomposition (EMD) [9], singular value decomposition (SVD) [10], principal component analysis (PCA) [11] and other methods were used to study the purification of the axis orbit in Ref. [9-11] and the remarkable results had been achieved.

Matching pursuit algorithm (MP algorithm) has been widely used in fields of fault diagnosis [12-19].

In this paper, a method to purify the axis orbit of large rotor based on matching pursuit algorithm is studied. Comparing the purification effect with PCA and harmonic wavelet, that the proposed algorithm has better applicability in the purification of the axis orbit of large-scale rotor test-bed.

\section{Basic Principles and Methods}

\subsection{Signal Sparse Decomposition and Matching Tracking Algorithm}

The specific process of MP algorithm is as follows [21]:
Firstly, select the core function $g_{r 0}$ that has the largest inner product with the signal to be decomposed as the optimal core function, the physical meaning of the criterion is that the projection of the signal to be decomposed on the original function is the largest. It means:

$$
\left|\left\langle y, g_{r 0}\right\rangle\right|=\sup _{r \in \Gamma}\left|\left\langle y, g_{r}\right\rangle\right|
$$

where $\left|\left\langle y, g_{r 0}\right\rangle\right|$ is the inner product of original signal $y$ and core function $g_{r 0}$. The original signal can be decomposed into two parts: the projection component on optimal core function $g_{r 0}$ and the residual component. It means:

$$
y=\left\langle y, g_{r 0}\right\rangle g_{r 0}+R^{1} y
$$

$R^{1} y$ is the residual signal after projection of original function on optimal kernel function. We repeat the above iterations for the residual components according to Eq. (2):

$$
R^{k} y=\left\langle R^{k} y, g_{r k}\right\rangle g_{r k}+R^{k+1} y
$$

where $g_{r k}$ satisfies the condition:

$$
\left|\left\langle R^{k} y, g_{r k}\right\rangle\right|=\sup _{r \in \Gamma}\left|\left\langle R^{k} y, g_{r k}\right\rangle\right|
$$

After decomposition for $M$ times, we can get that:

$$
y=\sum_{k=0}^{M-1}\left\langle R^{k} y, g_{r k}\right\rangle g_{r k}+R^{M} y
$$

where $R^{M} y$ is the reconstructed residual of original signal by using linear combination of $M$ kernel functions. 
We can prove that $\left\|R^{M} y\right\|$ decay to zero exponentially with the increase of $M$, so the signal can be decomposed by Eq. (6):

$$
y=\sum_{k=0}^{\infty}\left\langle R^{k} y, g_{r k}\right\rangle g_{r k}
$$

Under the premise of satisfying the error criterion, we can generally obtain the ideal result by using a linear combination of a few core functions. The combination rule is shown in Eq. (7):

$$
y \approx \sum_{k=0}^{M-1}\left\langle R^{k} y, g_{r k}\right\rangle g_{r k}
$$

where $M<<N, N$ is the data length of the original signal and the result fully reflects the idea of sparse decomposition.

\subsection{Axis orbit extraction by using MP algorithm}

The fault types of rotor system are usually determined by axis orbit. The main axis orbit determining the fault type of shafting consists of post-purified characteristic frequency like half harmonic $(0.5 \mathrm{X})$, rotational frequency (1X), second harmonic (2X). Generally, the vibration signal of a rotor contains noise signal, electromagnetic interference signal and high order harmonic frequencies, leading the axis orbit much complex. Furthermore, post-purified axis orbit emphasizing the primary factors such as $0.5 \mathrm{X}, 1 \mathrm{X}, 2 \mathrm{X}$ reflects the nature of the problem clearly.

Thus, when the axis orbit is extracted via MP Algorithm, the frequency domain characteristics need observation of Fast Fourier Transform (FFT), and then the characteristic frequency is extracted according to the frequency amplitude. Synthetic method of axis orbit based on MP algorithm is illustrated as follows:

Step 1: firstly, for a specific signal, non-DC component signal $y$ filtered out from original signal via FFT is obtained.

Step 2: non-dc component signal $y$ is transformed into FFT to observe the frequency domain character, and then is selected according to the characteristic frequency amplitude.

Step 3: over-complete dictionary set is constructed using cosine wave model, that is:

$$
g_{r_{k}}\left(t_{n}\right)=\cos \left(2 \pi f_{i} t_{n}+\varphi_{j}\right)
$$

where, $f_{i}$ is the frequency parameter, $i=1,2, \cdots M, \varphi_{j}$ is the phase parameter, $j=1,2, \cdots N, t_{n}$ is the $n$th sampling point. $n=1,2, \cdots L . L$ is the length of data. Two parameters can be evaluated evenly according to the given search precision, where the value of $f_{i}$ can be subdivided according to the neighborhood range of a characteristic frequency in the frequency domain, in other words, $f_{i}$ is subdivided into $M$ in the range of $[f-\Delta f f+\Delta f]$. Similarly, $\varphi_{j}$ can be divided into $N$ equal parts in the interval of $[-\pi, \pi]$. The atoms of the entire atom are $M N$. As $\left|g_{r}\right|=1$, amplitude is not needed to divide.

Step 4: atom $g_{r k}$ best matching to unbroken signal $y$ is selected from over-complete database. When the signal is sparse decomposed, original signal will reach the optimized match on a certain component. Since noise does not have the characteristics of a sinusoidal signal, projection on this component is close to zero. The optimized kernel function $g_{r k}$ is picked up on the largest inner product criterion. Expressed by the following formula:

$$
\left|\left\langle y, g_{r k}\right\rangle\right|=\sup _{r \in \Gamma}\left|\left\langle y, g_{r}\right\rangle\right|
$$

where $g_{r k}=\cos \left(2 \pi f_{k} t_{n}+\varphi_{k}\right)$ is the optimized kernel function and the component size of $y$ is $\alpha_{k}=<y, g_{r k}>$ on $g_{r k}$, thus, unbroken component is:

$$
R y=\alpha_{k} \cos \left(2 \pi f_{k} t_{n}+\varphi_{k}\right)
$$

Step 5: the axis orbit is synthesized according to the extracted characteristic frequency $1 \mathrm{X}, 2 \mathrm{X}$ or $3 \mathrm{X}$.

\section{Purifition of axis orbit}

\subsection{Test equipment}

The test data of axle orbit come from the large-scale rotor vibration test-bed independently, developed by the laboratory shown in Figure. 1. The terminal A is near the motor and the terminal B is far from the motor. Figure 1 shows the large-scale rotor test-bed, which is developed by our group.

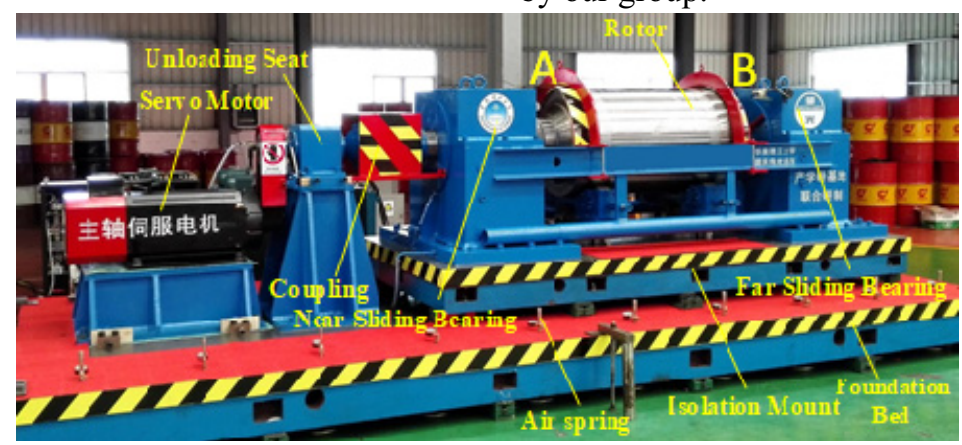

Figure 1. Large-scale rotor test-bed 
In order to monitor rotor working station in real time, two Kaman KD2306-1S eddy current displacement sensors are installed on individual side in vertical manners. The resolution is $0.01 \% \mathrm{FS}$ and the range is $0 \sim 2.5 \mathrm{~mm}$, shown in Figure 2. Experimental data is collected through LMS Test. Lab.

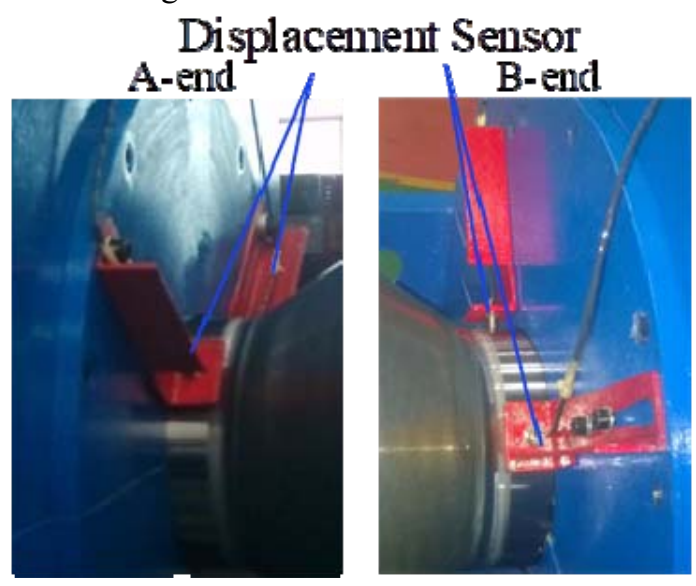

Figure 2. Installation of eddy current displacement sensor
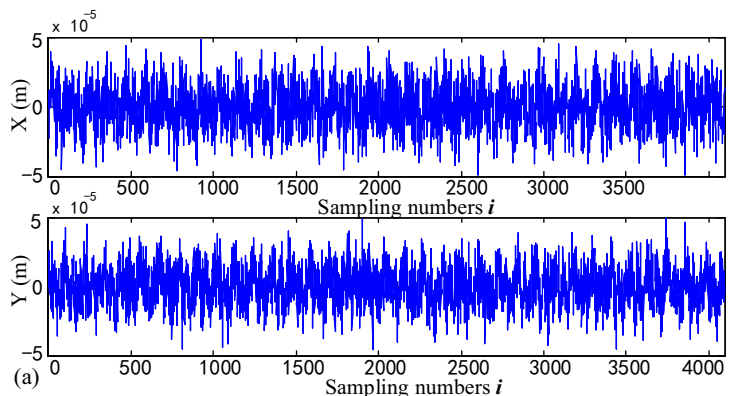

\subsection{Axis orbit extraction}

In this paper, a set of test data at both ends of A and B is analyzed. The sampling frequency is $2048 \mathrm{~Hz}$ and the sampling point is 4096. Collecting a set of working conditions near the motor end (terminal A) and the rotor speed is $1080 \mathrm{rpm}(18 \mathrm{~Hz})$ as the first analysis data in example 1. Collect the data of the remote motor end (terminal B) when the rotor speed is adjusted to 2760rpm $(46 \mathrm{~Hz})$ as the second analysis data in example 2 . In each of these embodiments, one set of data acts as the $\mathrm{X}$ axis and the other as the $\mathrm{Y}$ axis. The time-domain signals and amplitude spectra obtained by examples 1 and 2 are shown from Figure 3 to Figure 4.

Figure 3. Time-domain diagram of example 1 and example 2. (a) Time domain of example 1 displacement signal. (b) Time domain of example 2 displacement signal.
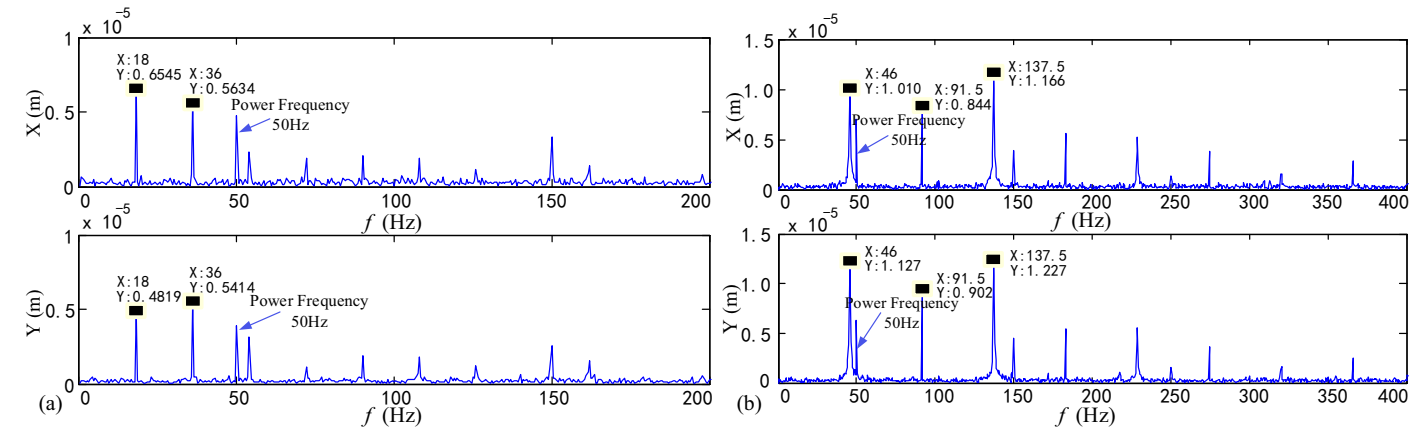

Figure 4. Frequency spectrum of raw signal of example 1 and example 2. (a) Frequency domain diagram of example 1. (b) Frequency domain diagram of example 2 .

From Figure 4, it can be seen that the original signals of two directions (X and $\mathrm{Y}$ ) are not only disturbed by ambient noise, but also have power frequency of $50 \mathrm{~Hz}$. Then, the axis orbit synthesized directly from the two displacement signals in Figure 3 is shown in Figure 5. It can be seen without any processing and the shape of the axis orbit can't be determined at all. 


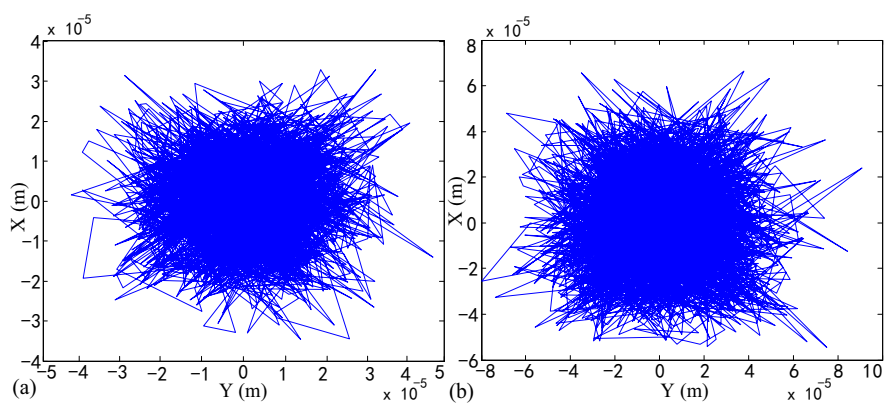

Figure 5. Axis orbit of raw signal. (a) Axis orbit of example 1. (b) Axis orbit of example 2.

From Figure 4 (a) and Figure 4 (b), we can see that the amplitude of the first double frequency is larger in example 1, and the amplitude of the first triple frequency is larger in example 2. Therefore, MP algorithm is used to extract the first double frequency and the first triple frequency to synthesize the axis orbit. When using the MP algorithm, the range is $\Delta f=2$ and the frequency step is 0.02 . At the same time, the phase step is $0.01 \mathrm{rad}$, and the results in frequency domain are shown in Figure 6 (a) and Figure 6 (b). At the same time, the 0.01 rad is
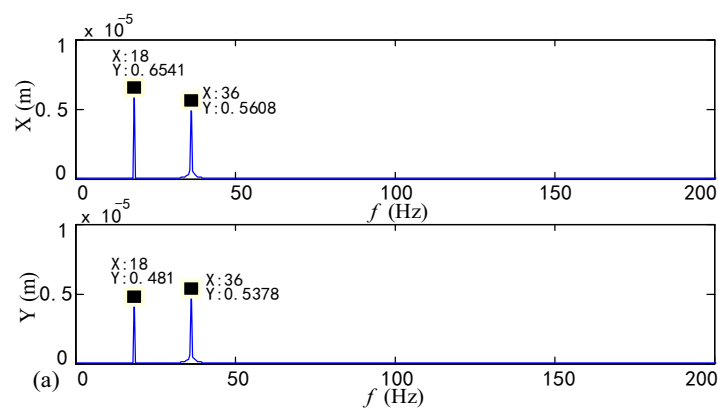

extracted from the phase step, and the results in frequency domain are shown in Figure 6 (a) and Figure 6 (b). It can be seen that the extraction effect of characteristic frequency is remarkable. Not only the noise interference is filtered, but also the interference of power frequency $50 \mathrm{~Hz}$ and other components is filtered. The extracted frequency amplitude is very close to the frequency doubling amplitude of the original signal (Figure 4), which indicates that the spectrum leakage is neglected.

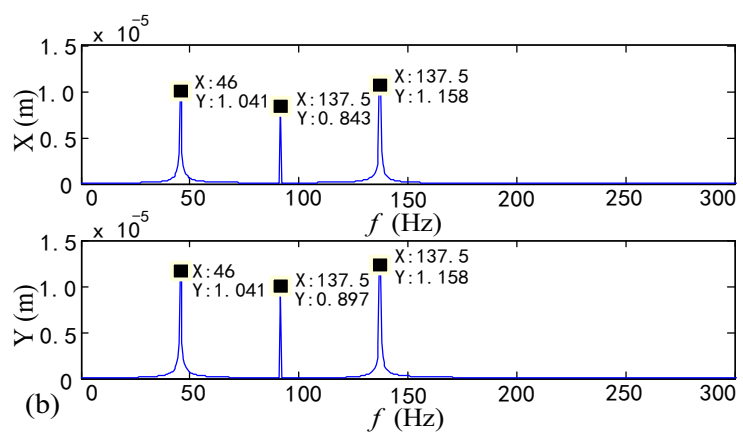

Figure 6. Frequency spectrums processed by MP algorithm. (a) Extraction of the first two frequency doubling of demo 1. (b) Extraction of the first three frequency doubling of demo 2.

Several common axis orbit shape and their causes of failure are shown in Table 1.

Table 1. Common rotor orbit and its cause of failure

\begin{tabular}{ccc}
\hline Fault factors & Axis orbit shape & Vibration dominant frequency \\
\hline Rotor unbalance & Ellipse & Rotating frequency $(1 \mathrm{X})$ \\
Rotor misalignment & Banana shape or Outer 8 shape & $1 \mathrm{X}$, second harmonic $(2 \mathrm{X})$ \\
Oil whirl & Inner 8 shape & half harmonic $(1 / 2 \mathrm{X})$ \\
Rub-impact & Petal shape & $1 / 2 \mathrm{X}, 1 / 3 \mathrm{X}, 3 \mathrm{X}$ \\
\hline
\end{tabular}

Using the signals purified in Figure 6 (a) and Figure 6 (b), the axis orbits are synthesized as shown in Figure 7 and Figure 8. It can be seen that the axis orbits are banana shape and petal shape respectively, which shows that there is a phenomenon of misalignment on the near motor end (terminal A) in example 1 and a phenomenon of rub-impact between static and dynamic parts on the remote motor end (terminal B) in example 2. After using MP algorithm, the fault characteristics of the rotor system can be clearly obtained, thus the fault diagnosis of large rotor system can be accurately realized. 

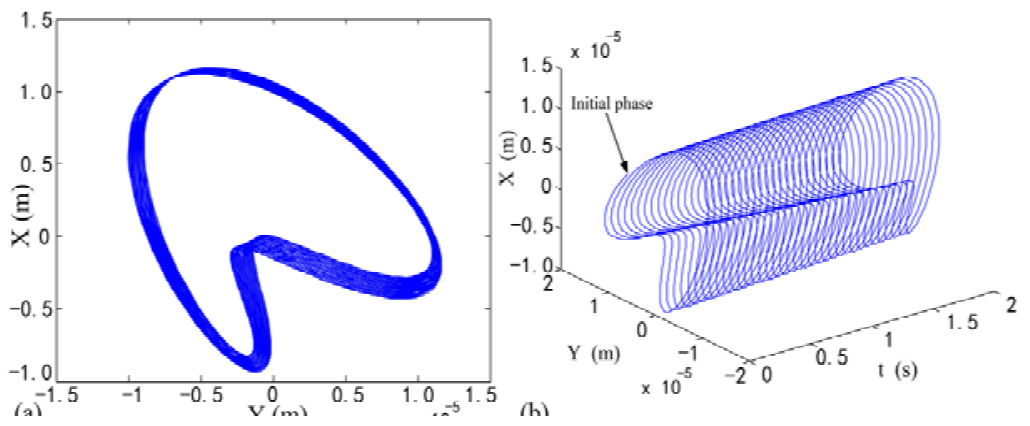

Figure 7. Axis orbit of data synthesis in Figure. 6 (a). (a) 2D axis orbit (b) 3D axis orbit
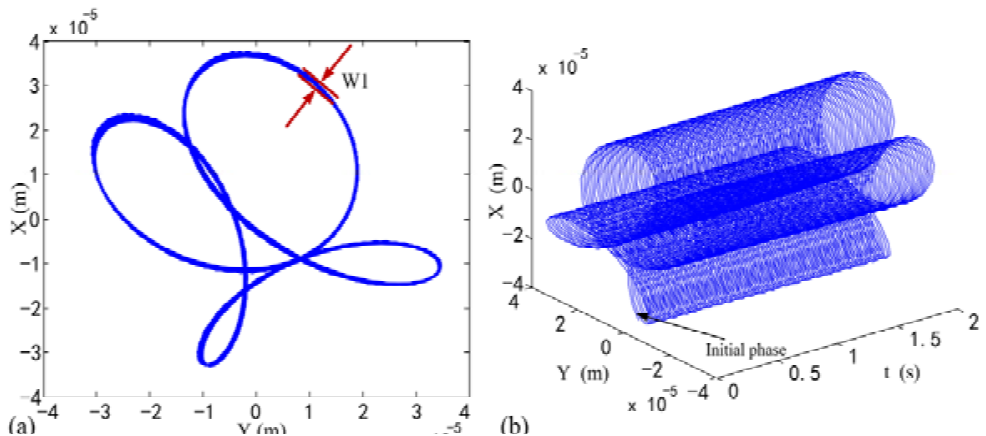

Figure 8. Axis orbit of data synthesis in Figure. 6 (b). (a) 2D axis orbit (b) 3D axis orbit

Through analysis, we can see that the causes of the failure are as follows: In example 1, because of the low speed of the rotor, the supporting oil film can't be fully established. There is a certain deviation between the rotor and the coupling, resulting in the phenomenon of misalignment. In example 2 , because the test bench is supported by tilting pad hydrodynamic oil film bearings, when the oil pressure of hydraulic station is insufficient, the rotor $(5000 \mathrm{Kg}$ ) can't float completely (oil film thickness is insufficient), resulting in the collision between the rotor shaft diameter and the bearing bush.

From Figure 7 (b) and Figure 8 (b), we can see that when the axis orbit changes with time, the oscillation is small and the amplitude is stable. Because the phase requirement for the purification of the axis orbit is high, if the phase of the $\mathrm{X} / \mathrm{Y}$ signal is deviated, the synthesized axis orbit will be distorted greatly. The phase accuracy of MP algorithm increases with the decrease of phase step size, but it also increases the calculation cost. In this paper, the phase step size of $0.01 \mathrm{rad}$ can meet the requirements.
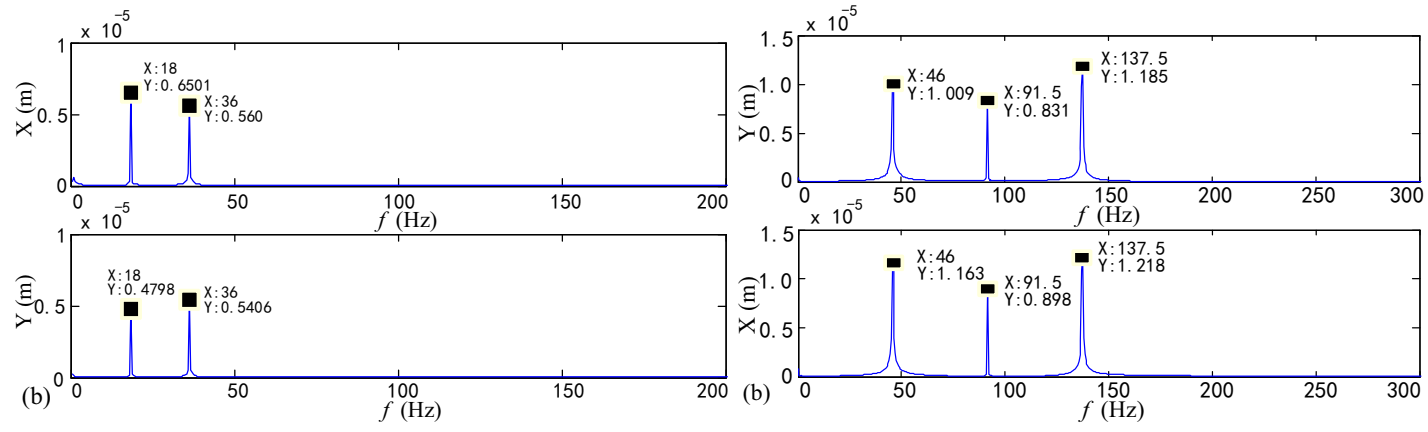

Figure 9. Frequency spectrums processed by PCA algorithm. (a) Extraction of the first two frequency doubling of demo 1. (b) Extraction of the first three frequency doubling of demo 2.

The axis orbit of signal synthesis filtered by PCA

algorithm, shown in Figure 10, shaping of banana and 
plum blossom, is consistent with the outline and trend of MP algorithm, but the result of MP algorithm is not clear. For example, in example 2 , the orbit width of Figure

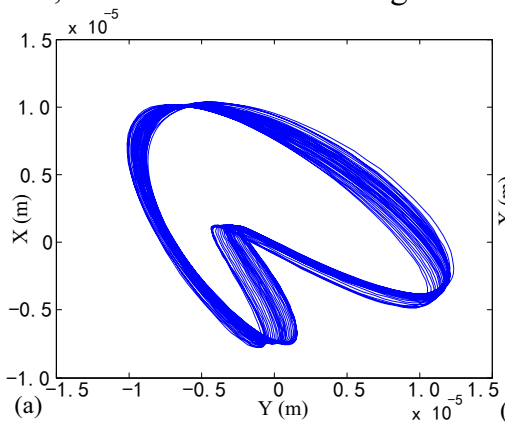

10 (b) is larger than that of Figure $8(a)$, i.e. W2 $>$ W1, which indicates that the orbit of MP algorithm has less divergence.

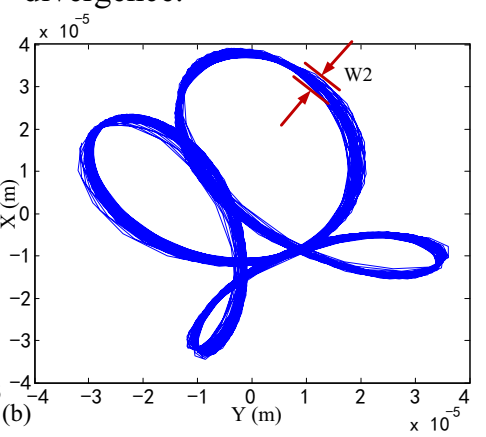

Figure 10. Axis orbit obtained by PCA algorithm. (a) Axis orbit of example 1. (b)Axis orbit of example 2.

\subsubsection{Comparison with harmonic wavelet algorithm}

Harmonic wavelet algorithm is an improved wavelet algorithm, which can extract the fundamental frequency and its frequency doubling signal easily in the purification of the axis orbit of rotating test-bed. It is one of the commonly used signal processing methods in rotating test-bed.

Harmonic wavelet algorithm is used to purify the orbit of shaft center in case 1 and case 2. Example 1 is carried out by 8 -level decomposition. The bandwidth is

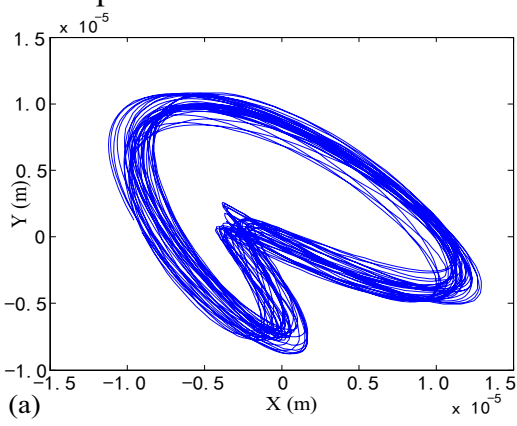

(a)

Figure 11. Axis orbit obtained by harmonic wavelet method.

The orbit of shaft center synthesized by characteristic frequency, which uses harmonic wave to purity, is shown in Figure 11. Compared with Figure 10, it is clear that the PCA algorithm is more effective and the orbit is more compact. This is due to much noise in the frequency band when harmonic wavelet is extracted.

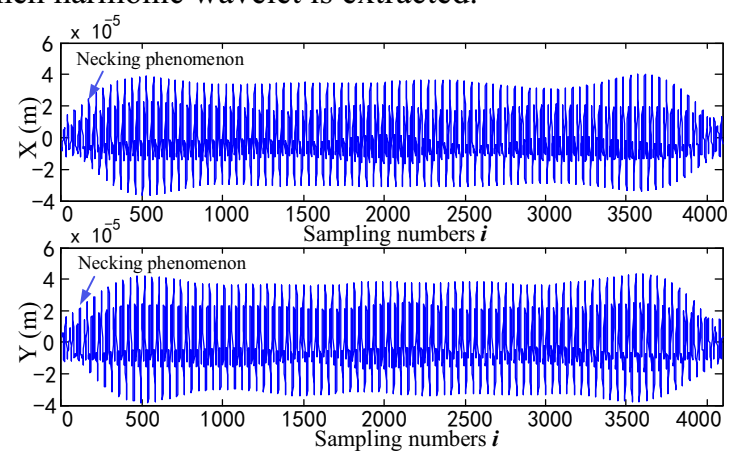

Figure 12. Time domain of harmonic wavelet processing example 2 .
$4 \mathrm{~Hz}$, the fundamental frequency is $18 \mathrm{~Hz}$ and the doubling frequency is $36 \mathrm{~Hz}$, which fall in the frequency bands of $[16,20]$ and $[34,36]$ respectively. Similarly, for example 2, 8-level decomposition is also performed, with bandwidth of $4 \mathrm{~Hz}$, fundamental frequency of $46 \mathrm{~Hz}$, double frequency of $91.5 \mathrm{~Hz}$ and triple frequency of $137.5 \mathrm{~Hz}$, which fall in the frequency bands of $[44,48]$, $[88,92]$ and $[136,140]$ respectively. It can be seen in example 2, the fundamental frequency $46 \mathrm{~Hz}$ can be separated from the power frequency $50 \mathrm{~Hz}$, and the power frequency interference can be effectively removed.

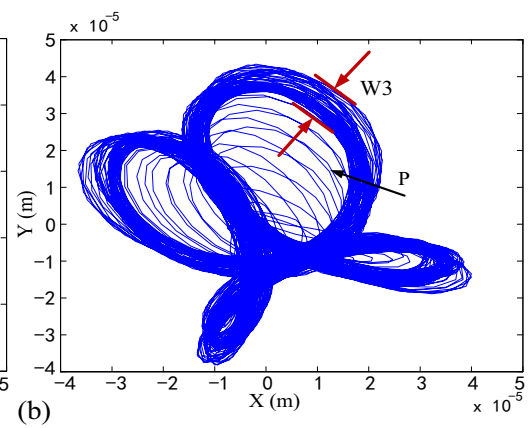

(b)

. (a) Axis orbit of example 1. (b)Axis orbit of example 2.

At the same time, we can see from Figure 11(b) that hyperbola appears at the $\mathrm{P}$ position of the axis orbit. However, there is no such phenomenon in the MP algorithm and PCA algorithm. The analysis is as follows: this is because the harmonic wavelet algorithm is limited by the indeterminancy principle, the time domain resolution $\sigma_{t}$ and frequency domain resolution $\sigma_{\omega}$ can not be arbitrarily high. It can be expressed as an expression: $\sigma_{t}^{2} \cdot \sigma_{\omega}^{2} \geq 1 / 4$. When the resolution in frequency domain is small, the resolution in time domain will be reduced, resulting in the phenomenon of "closing" at both ends of the time domain map of characteristic frequency (Figure 16), which makes the appearance of hyperbolic curve in the center of the orbit of shaft center. If the bandwidth of frequency domain is increased and the resolution of time domain is improved, the noise in frequency band will increase again, which will cause the curve divergence, that means W3 (Figure 12) will increase continuously. It is precisely because of 
this contradiction that harmonic wavelet algorithm is difficult to develop in characteristic frequency extraction.

Finally, the compound fault analysis of the test-bed is carried out. In practical working conditions, the rotor orbit is usually the result of multiple fault combinations. In that way, the MP algorithm is used to extract the synthetic axis trajectories of $1 \mathrm{X}$ and $2 \mathrm{X}$ from the experimental data in example 2 (Figure 4 (b)). The results are shown in Figure 13. It can be seen that the axis orbit is the shape of banana and the depression is to a lesser degree indicating that there is a slight misalignment near the power-driven machine end of the test-bed. The existence of these two typical faults also shows that there are multiple faults in large-scale rotor test-bed.

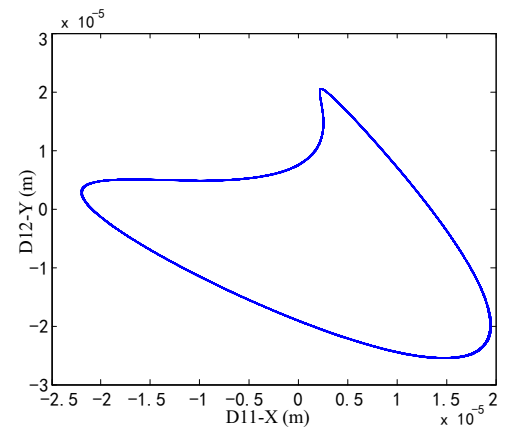

Figure 13. Axis orbit of the first two frequency syntheses by MP algorithm

\section{Conclusions}

This article uses MP algorithm to extract axis orbit, in order to realize the purification of rotor axis orbit in large bearing test stand. The conclusions are as follows:

(1) The method of using MP algorithm to extract axis orbit can eliminate the interference of random noise.

(2) MP algorithm, proves the superiority of this algorithm in the purification of axis orbit.

(3) It can be seen that there are multiple faults in the large-scale rotor system, so as to understand the operation status of the test-bed.

\section{Acknowledgments}

This work is supported by the following three projects.

1. 2018 Guangdong Science and Technology Plan Project All Direct Drive High Speed and High Precision Roll Compound CNC Machine Tool Equipment (Grand No. 2019B090918003)

2. Development and Application of Environment-friendly Intelligent Equipment for Hull Abrasive Water Jet to Remove Paint and Rust (GDNRC [2020] 030)

3. National Natural Science Foundation of China (NSFC, Grant No. 51875205)

\section{Reference}

1. Shi, D.F., Wang W.J., Unsworth P.J., Qu L.S. (2005) Purification and feature extraction of shaft orbits for diagnosing large rotating machinery. J. Sound Vib. 279(3-5):581-600.

2. Yang W. and Tavner P.J. (2009) Empirical mode decomposition, an adaptive approach for interpreting shaft vibratory signals of large rotating machinery. J. Sound Vib. 321(3-5):1144-1170.

3. Wu F. and Qu L. (2008) An improved method for restraining the end effect in empirical mode decomposition and its applications to the fault diagnosis of large rotating machinery. J. Sound Vib. 314(3-5):586-602.

4. Guo M.J., Li W.G., Yang Q.J. (2020) Sparse Algorithm-Based Purification of Multi-Condition Axis orbit of Large Rotor. Journal of South China University of Technology (Natural Science Edition), 48(04):45-53.

5. Zhang D.B., Fang X., Wu H.C., Zhang X.Y., He S.X., Liu C.W. (2019) Recognition classification based on $\mathrm{Hu}$ moment invariants and imperial competitive algorithm for axis orbit of magnetic bearing-rotor system. Personal and Ubiquitous Computing, (prepublish).

6. Zhang W.B., Zhou X.J. and Lin Y. (2010) Refinement of rotor center's orbit by a harmonic window method. J. Vib. Meas. Diag. 30(01): 87-90.

7. Kougioumtzoglou I.A. and Spanos P.D. (2013) An identification approach for linear and nonlinear time-variant structural systems via harmonic wavelets. Mech. Syst. Signal Pr. 37(1): 338-352.

8. Zhang W.B., Zhou X.J., Yang X.Y. and Shen L. (2010) Purification of rotor axis's orbit based on morphological wavelet. J. ZheJiang Univ. 44(8):1449-1453.

9. Wu, F.J. and Qu, L.S. (2008) An improved method for restraining the end effect in empirical mode decomposition and its applications to the fault diagnosis of large rotating machinery. J. Sound Vib. 314(3): 36-50.

10. Liu W.G. (2016) Research on method of purification of shaft orbit based on singular value decomposition. Tech. Acou. 5(35): 30-35.

11. Li W.G. and Li Z. (2017) A PCA-based feature frequency extraction method. 11169833.0, 2017-12-15.

12. Wang H.C., Chen J. and Dong G.M. (2014) Weak fault feature extraction of rolling bearing based on minimum entropy de-convolution and sparse decomposition. J. Vib. Control20(8): 1148-1162.

13. Peng F.Q., Yu D.J. and Jian L. (2010) Sparse signal decomposition method based on multi-scale chirplet. J. Vib. Engtehnol. 23(3):333-338.

14. Sun J., Su Y.X. (2020) Weak Ultrasound echo extraction optimized by adaptive sparse decomposition. Laster \& Optoelectronics Progress: 1-7[08-24].

15. Wang H.C., Du W.L. (2020) A new K -means singular value decomposition method based on self-adaptive matching pursuit and its application in 
fault diagnosis of rolling bearing weak fault. International Journal of Distributed Sensor Networks, $16(5)$.

16. Yi Z.K., Shao J. and Pierre V. (2006) MP based Signal sparse decomposition with FFT. J. Elec. Info. Tech. 28(04): 614-618.

17. Yan B.K., Zhou F.X., Zhang RH (2016) Extraction of Twin Impulses Based on Sparse Decomposition. Journal of Vibration, Measurement \& Diagnosis 36(02): 301-308+402.

18. Lin J., Zhao Z.D. and Sun S.Q. (2017) Algorithm for finger-ECG signal identity recognition based on sparse coding. Tran. Micr. Tech. 36(02):142-145.

19. Li H.L. (2016). Study on rotor faults diagnosis base on matching pursuit and information entropy. North University of China. 